\title{
Plasma heating and generation of energetic D ions with the 3-ion ICRF + NBI scenario in mixed H-D plasmas at JET-ILW
}

Kazakov, Ye O.; Nocente, M.; Mantsinen, M. J.; Ongena, J.; Baranov, Y.; Craciunescu, T.; Dreval, M.; Dumont, R.; Eriksson, J.; Garcia, J.

Total number of authors:

21

Published in:

Nuclear Fusion

Link to article, DOI:

10.1088/1741-4326/ab9256

Publication date:

2020

Document Version

Peer reviewed version

Link back to DTU Orbit

Citation (APA):

Kazakov, Y. O., Nocente, M., Mantsinen, M. J., Ongena, J., Baranov, Y., Craciunescu, T., Dreval, M., Dumont, R., Eriksson, J., Garcia, J., Giacomelli, L., Kiptily, V. G., Kirov, K. K., Meneses, L., Nabais, F., Nave, M. F. F., Salewski, M., Sharapov, S. E., Stancar, Z., ... Weisen, H. (2020). Plasma heating and generation of energetic D ions with the 3-ion ICRF + NBI scenario in mixed H-D plasmas at JET-ILW. Nuclear Fusion, 60(11), [112013 ]. https://doi.org/10.1088/1741-4326/ab9256

\section{General rights}

Copyright and moral rights for the publications made accessible in the public portal are retained by the authors and/or other copyright owners and it is a condition of accessing publications that users recognise and abide by the legal requirements associated with these rights.

- Users may download and print one copy of any publication from the public portal for the purpose of private study or research.

- You may not further distribute the material or use it for any profit-making activity or commercial gain

- You may freely distribute the URL identifying the publication in the public portal 


\title{
Plasma heating and generation of energetic $D$ ions with the 3-ion ICRF + NBI scheme in mixed H-D plasmas at JET-ILW
}

\author{
Ye.O. Kazakov ${ }^{1}$, M. Nocente ${ }^{2,3}$, M.J. Mantsinen ${ }^{4,5}$, J. Ongena ${ }^{1}$, Y. Baranov ${ }^{6}$, \\ T. Craciunescu ${ }^{7}$, M. Dreval ${ }^{8}$, R. Dumont ${ }^{9}$, J. Eriksson ${ }^{10}$, J. Garcia ${ }^{9}$, L. Giacomelli ${ }^{3}$, \\ V.G. Kiptily ${ }^{6}$, K. Kirov ${ }^{6}$, L. Meneses ${ }^{11}$, F. Nabais ${ }^{11}$, M.F.F. Nave ${ }^{11}$, M. Salewski ${ }^{12}$, \\ S.E. Sharapov ${ }^{4}$, Ž. Štancar ${ }^{13}$, J. Varje ${ }^{14}$, H. Weisen ${ }^{15}$ and JET Contributors*
}

${ }^{1}$ Laboratory for Plasma Physics, LPP-ERM/KMS, Brussels, Belgium

${ }^{2}$ Dipartimento di Fisica, Università di Milano-Bicocca, Milan, Italy

${ }^{3}$ Institute for Plasma Science and Technology, National Research Council, Milan, Italy

${ }^{4}$ Barcelona Supercomputing Center (BSC), Barcelona, Spain

${ }^{5}$ ICREA, Barcelona, Spain

${ }^{6}$ CCFE, Culham Science Centre, Abingdon, UK

${ }^{7}$ National Institute for Laser, Plasma and Radiation Physics, Bucharest, Romania

${ }^{8}$ National Science Center 'Kharkiv Institute of Physics and Technology', Kharkiv, Ukraine

${ }^{9}$ CEA, IRFM, Saint-Paul-lez-Durance, France

${ }^{10}$ Department of Physics and Astronomy, Uppsala University, Uppsala, Sweden

${ }^{11}$ IST, Universidade de Lisboa, Lisbon, Portugal

${ }^{12}$ Technical University of Denmark, Kgs. Lyngby, Denmark

${ }^{13}$ Jožef Stefan Institute, Ljubljana, Slovenia

${ }^{14}$ Aalto University, Finland

${ }^{15}$ EPFL, Swiss Plasma Center (SPC), Lausanne, Switzerland

* See the author list of "Overview of the JET preparation for Deuterium-Tritium

Operation” by E. Joffrin et al., Nucl. Fusion 59, 112021 (2019)

\begin{abstract}
Dedicated experiments were conducted in mixed H-D plasmas in JET to demonstrate the efficiency of the 3-ion ICRF scheme for plasma heating, relying on injected fast NBI ions as resonant ion species. Strong core localization of the RF power deposition in the close vicinity of the ion-ion hybrid layer was achieved, resulting in an efficient plasma heating, generation of energetic D ions, strong enhancement of the neutron rate and observation of Alfvénic modes. A consistent physical picture that emerged from a range of fast-ion measurements at JET, including neutron and gamma-ray measurements, a high-energy neutral particle analyzer and MHD mode localization analysis, is presented. The possibility to moderate the fast-ion energies with the ratio $P_{\mathrm{ICRF}} / P_{\mathrm{NBI}}$ and the choice of the NBI injectors is demonstrated. An outlook of possible applications of the 3-ion schemes, including a recent example of its use in mixed D- ${ }^{3} \mathrm{He}$ plasmas in JET and promising schemes for D-T plasmas, are presented.
\end{abstract}




\section{Introduction}

Auxiliary heating systems are essential for a fusion reactor to reach high ion temperatures for thermonuclear fusion. Radio frequency plasma heating with waves in the ion cyclotron range of frequencies (ICRF) is a well-established technique that has been successfully applied in present-day tokamaks and is foreseen for ITER [1-4]. ICRF heating relies on the excitation of fast magnetosonic waves (FW) that can undergo a large variety of collisionless interactions in the plasma, both with ions and electrons. Matching locally the Dopplershifted wave frequency with the particle's cyclotron frequency or its harmonics is one of the pre-requisites for strong wave-particle interactions to occur

$$
\omega=n \omega_{\mathrm{cs}}+k_{\|} \mathrm{V}_{\|, \mathrm{s}}(n=0, \pm 1, \pm 2, \ldots) .
$$

Here, $\omega$ is the angular wave frequency, $\omega_{\mathrm{cs}}=q_{\mathrm{s}} B / m_{\mathrm{s}}$ is the local cyclotron frequency of particles with charge $q_{\mathrm{s}}$ and mass $m_{\mathrm{s}}, k_{\|}$and $\mathrm{v} \|, \mathrm{s}$ are the components of the wave number and particle velocity parallel to the confining magnetic field. In turn, Eq. (1) determines the parallel velocities for resonant ions

$$
\mathrm{V}_{\|, \mathrm{i}}=\left(\omega-n \omega_{c s}\right) / k_{\|} .
$$

For thermal ions with low $\mathrm{v}_{\|}$, this condition can be locally fulfilled close to the ion cyclotron resonance layers, where $\omega \approx n \omega_{\text {ci. }}$.

The condition given by Eq. (2) alone is not sufficient for efficient wave absorption by the resonant ions. As the electric field of excited fast waves can be decomposed as a sum of two components, $E_{+}$(left-hand polarized, rotating in the direction of the ions) and $E_{-}$(right-hand polarized, rotating in the opposite direction), it is important to note that thermal ions (as well as moderately energetic ions with energies in the range of a few hundred $\mathrm{keV}$ ) interact mainly with the left-hand polarized RF component $E_{+}$[5]. The ratio $E_{+} / E_{-}$is a local quantity in a plasma, and its spatial profile is primarily determined by the chosen plasma composition, namely the number of ion species and their concentrations, $X_{\mathrm{i}}=n_{\mathrm{i}} / n_{\mathrm{e}}$ ( $n_{\mathrm{e}}$ is electron density and $n_{\mathrm{i}}$ is the ion density). To illustrate the importance of local wave polarization, we note that fundamental $(n=1)$ ICRF heating of single-ion plasmas is inefficient due to the fact that the $E_{+}$component almost vanishes at $\omega \approx \omega_{\text {ci }}[6]$.

A review of traditionally used ICRF scenarios is given, e.g., in [5-7]. Out of these, minority heating schemes became the workhorse scenarios for ICRF heating in fusion research. In short, these scenarios can be realized in plasmas, including at least two ion populations with different $\omega_{\text {ci }}$. The concentration of one of the ion species (the so-called minority species) has to be rather small to guarantee efficient wave damping in the region of its ion cyclotron resonance $(n=1)$. The operational range for this efficient ICRF scheme is usually limited by minority concentrations of a few $\%$. Under these conditions, the ion-ion hybrid (IIH) pair, which is characterized by a local enhancement of the $E_{+}$component, is located close to the cyclotron resonance of the minority ions and facilitates damping of fast waves by minority ions. At higher minority concentrations, the efficiency of minority ion damping is reduced and ICRF heating via mode conversion becomes dominant $[8,9]$. This is because the ion-ion hybrid layer moves away from the minority cyclotron resonance layer and thermal ions can no longer fulfil Eq. (2) in the region with enhanced $E_{+}$polarization. In this case, the incoming fast wave undergoes a transformation into short wavelength modes and then, in a second step, the mode converted waves are absorbed usually by the electrons in the plasma. The localization of RF heating in this heating scenario is determined by the location of the ion-ion hybrid layer in the plasma and can be externally controlled by varying the plasma composition [10]. 
Recently, novel '3-ion' ICRF schemes have been proposed and successfully tested experimentally as a solution to achieve dominant ion absorption in such mixed plasmas instead of mode conversion heating $[11,12]$. This technique relies on extending the plasma composition beyond two thermal ion species only and providing an additional ('third') population of ions that fulfill Eq. (2) and resonantly absorb RF power close to the ion-ion hybrid layer(s) in mixed plasmas. Two possibilities to do so follow immediately from Eq. (1).

As a first option, one can use ions with an intermediate charge-to-mass ratio as a resonant minority, $(Z / A)_{2}<(Z / A)_{3}<(Z / A)_{1}$, e.g., ${ }^{3} \mathrm{He}$ ions for heating $\mathrm{H}-\mathrm{D}$ and $\mathrm{H}-{ }^{4} \mathrm{He}$ mixed plasmas, confirmed in 3-ion ICRF experiments on JET, Alcator C-Mod and AUG tokamaks $[13,14]$. These experiments demonstrated the high-efficiency of ICRF for plasma heating, not only at ${ }^{3} \mathrm{He}$ minority concentrations of $\sim 1-2 \%$, but equally at much lower ${ }^{3} \mathrm{He}$ concentrations of a few per mille (\%) only. 3-ion ICRF schemes also allow the use of impurity ions to optimize the deposition of ICRF power in fusion plasmas. As an example, intrinsic ${ }^{9} \mathrm{Be}$ impurities have been predicted to absorb efficiently ICRF power in D-T plasmas of JET-ILW and ITER, resulting in strong bulk ion heating [15].

The second option to realize the 3-ion ICRF scheme in mixed plasmas (i.e., achieving dominant fast wave absorption by ions rather than mode conversion heating) relies on using fast ions with large parallel velocities as a resonant 'third' population $[12,16]$. Fast ions with a sufficiently large Doppler shift, $k\|\mathrm{~V}\|$ can resonate in the vicinity of the ion-ion hybrid layer through the second term in Eq. (1). In this case, the $(Z / A)$ value of the resonant fast ions does not necessarily have to differ from that of the non-resonant ions, $(Z / A)_{1}$ and $(Z / A)_{2}$. A natural choice for the realization of the Doppler-shifted version of the 3-ion ICRF scheme are fast ions injected by neutral beam injection (NBI). Under these conditions, we have effectively a 2-stage particle accelerator: fast ions from by NBI system (stage \#1) efficiently absorb RF power at the ion-ion hybrid layer and are further accelerated to high energies by the injected fast waves by ICRF (stage \#2). Note that the same physics applies to energetic ions that are products of fusion reactions. Absorption of ICRF power by alpha particles in D-T plasmas is well known and is usually considered as a parasitic effect to be avoided.

A set of dedicated 3-ion ICRF + NBI experiments was performed in mixed H-D [12] (and very recently in $\mathrm{D}^{3} \mathrm{He}$ ) plasmas in JET-ILW proving the high-efficiency of plasma heating and fast-ion generation with this novel scheme. The successful realization of this scheme extends the application of ICRF beyond heating of mixed plasmas only. In particular, this scheme is a useful tool for the following studies at JET:

- maximizing beam-target fusion, in particular in D- ${ }^{3} \mathrm{He}$ and D-T plasmas;

- generating fast ions and studying fast-ion driven MHD instabilities, even at rather high plasma densities;

- studying energy transport at ITER-relevant heating conditions, when electron heating from MeV-range fast ions dominates.

This paper is organized as follows. The details of the experimental conditions in JET, in particular the selected plasma composition in mixed H-D plasmas and sensitivity to the choice of the NBI injectors are presented in section 2. Observations with a range of fast-ion diagnostics are described in section 3, confirming the acceleration of fast D-NBI ions to higher energies with ICRF waves. An outlook of possible applications of the 3-ion ICRF schemes, including a recent example of its use in mixed $\mathrm{D}-{ }^{3} \mathrm{He}$ plasmas in JET and promising schemes for D-T plasmas, are given in section 4. Finally, summary and conclusions are presented in section 5 . 


\section{Summary of the 3-ion ICRF + NBI experiments in H-D plasmas at JET-ILW}

To demonstrate experimentally ICRF absorption by fast beam particles in the vicinity of the ion-ion hybrid layer, a number of dedicated pulses were performed in the past Hydrogen campaign in JET-ILW. Figure 1 shows an overview of JET pulse \#91256, first discussed in [12] and later also in [14, 16-18], which is the focus of the analysis reported in this paper. The discharge has three different phases of applied ICRF-to-NBI power ratio, $P_{\mathrm{ICRF}} / P_{\mathrm{NBI}}$. The pulse starts with an NBI-only phase $\left(P_{\mathrm{NBI}} \approx 3.5 \mathrm{MW} ; t=7.5-9.0 \mathrm{~s}\right)$, followed by two phases of combined ICRF $+\mathrm{NBI}$ heating with $P_{\mathrm{ICRF}} / P_{\mathrm{NBI}} \approx 0.7 \quad\left(P_{\mathrm{ICRF}} \approx 2.5 \mathrm{MW}\right.$, $\left.P_{\mathrm{NBI}} \approx 3.5 \mathrm{MW}\right)$ and $P_{\mathrm{ICRF}} / P_{\mathrm{NBI}} \approx 0.3\left(P_{\mathrm{ICRF}} \approx 1.3 \mathrm{MW}, P_{\mathrm{NBI}} \approx 4.9 \mathrm{MW}\right)$. Tangential NBI injectors $(\lambda=\mathrm{v} \| / \mathrm{v} \approx 0.62)$ with a maximum injection energy of $100 \mathrm{keV}$ were used. Up to $2.5 \mathrm{MW}$ of ICRF power was delivered at a frequency $f=25 \mathrm{MHz}$, using dipole phasing (symmetric $k_{\|}$-spectrum with dominant $n_{\text {tor }}= \pm 27$ [8], $k_{\|} \approx n_{\text {tor }} / R$ ). As can be seen in Fig. 1, the central electron temperature, the period of the sawtooth, the neutron rate and gamma-ray emission are strongly increased when ICRF power was applied on top of the NBI power. Moreover, the increase also depends on the ratio $P_{\mathrm{ICRF}} / P_{\mathrm{NBI}}$.

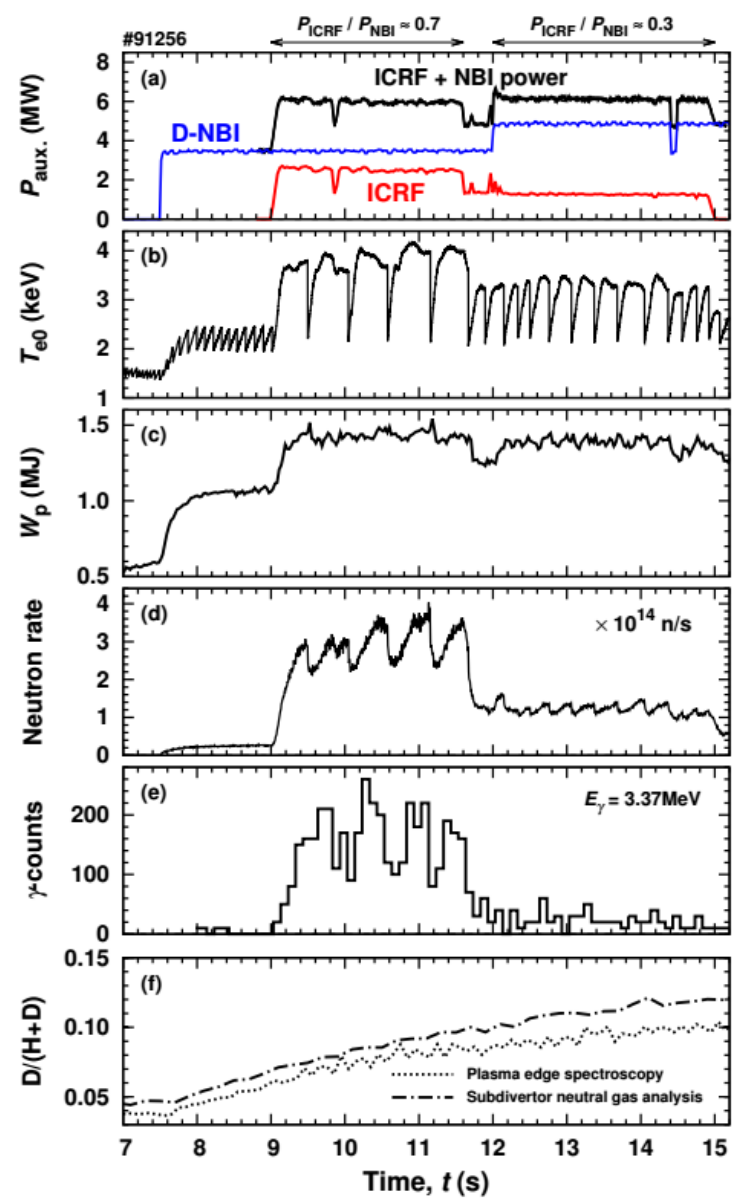

Figure 1. Overview of JET pulse \#91256, in which the 3-ion D-( $\left.\mathrm{D}_{\mathrm{NBI}}\right)-\mathrm{H}$ scheme was applied for heating mixed H-D plasma (L-mode, $B_{0} \approx 2.9 \mathrm{~T}, I_{\mathrm{p}} \approx 2 \mathrm{MA}, n_{\mathrm{e} 0} \approx 4 \times 10^{19} \mathrm{~m}^{-3}$, $X[\mathrm{H}] \approx 85-90 \%, X[\mathrm{D}] \approx 10-15 \%, f=25 \mathrm{MHz}$, dipole phasing). (a) Auxiliary heating power (NBI, ICRF and ICRF+NBI); (b) Central electron temperature; (c) Plasma stored energy; (d) Neutron rate; (e) Number of gamma-ray counts at $E_{\gamma}=3.37 \mathrm{MeV}$, produced in nuclear reactions between fast $\mathrm{D}$ ions and ${ }^{9} \mathrm{Be}$ impurities (threshold $\mathrm{D}$ energy, $E_{\mathrm{D}} \approx 0.5 \mathrm{MeV}$ ); (f) Evolution of the edge isotopic ratio $\mathrm{D} /(\mathrm{H}+\mathrm{D})$, measured by two different methods. 
The location of the ion cyclotron resonances for thermal $\mathrm{D}$ and $\mathrm{H}$ ions in pulse \#91256 is shown in Fig. 2(a): $R_{\mathrm{IC}}(\mathrm{D}) \approx 2.6 \mathrm{~m}$ (off-axis at the high magnetic field side) and $R_{\mathrm{IC}}(\mathrm{H}) \approx 5.2 \mathrm{~m}$ (outside the plasma). The choice of the off-axis location of the $\mathrm{D}$ cyclotron resonance is intentional. Indeed, as follows from the discussion in the introduction of the paper, wave absorption and plasma heating are expected to have a maximum in the vicinity of the ion-ion hybrid pair, where the $E_{+}$polarization is particularly favorable for the damping of fast waves on ions. Figure 2(a) also shows the computed location of the IIH pair at $X[\mathrm{D}]=15 \%$ and $30 \%$, assuming $n_{\text {tor }}=27$ and the presence of $0.5 \%$ of intrinsic beryllium impurities in the plasma. We note that the position of the ion-ion hybrid pair in H-D plasmas can be approximated as $R_{\mathrm{IIH}} \approx R_{\mathrm{IC}}(\mathrm{D}) \times(1+X[\mathrm{D}])$. As a result, deuterium concentrations of $10-15 \%$ are sufficient to position the ion-ion hybrid layer close to the plasma core.
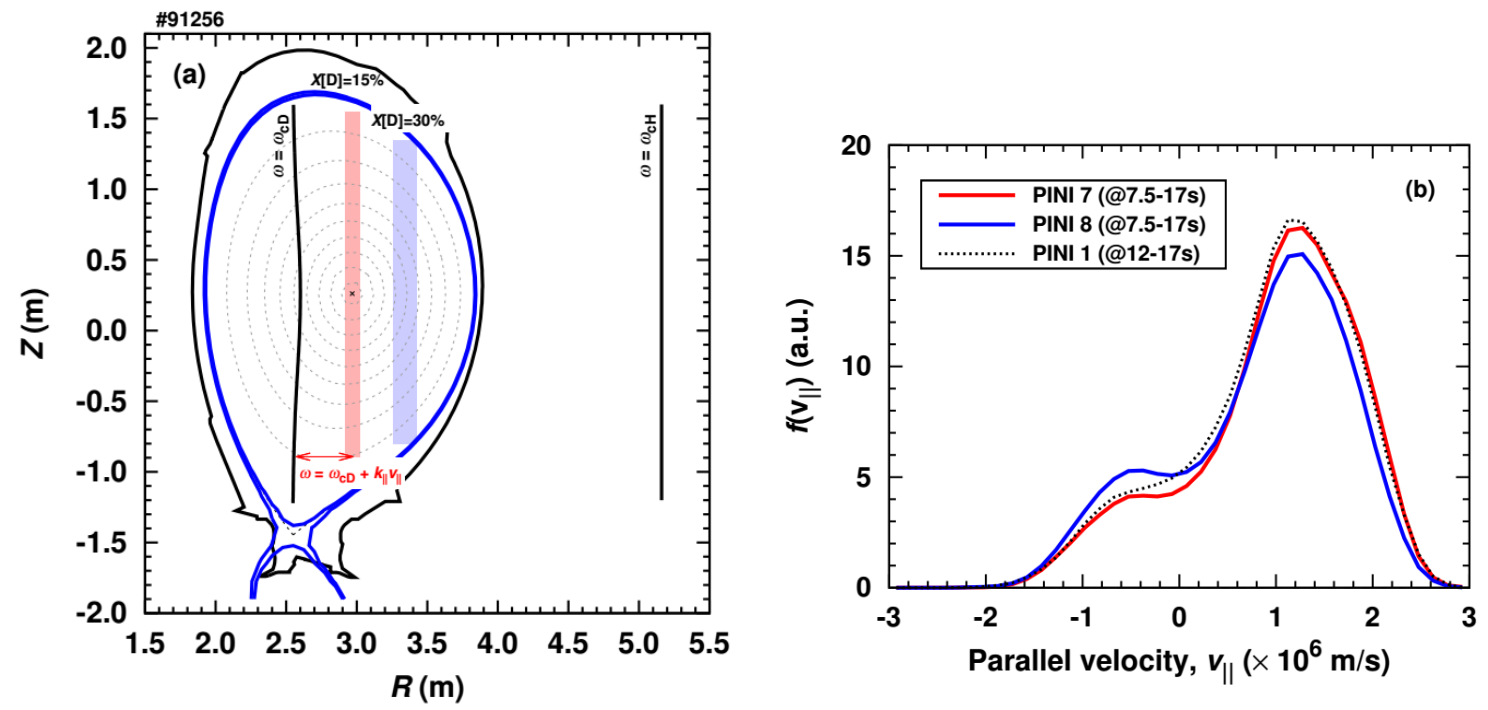

Figure 2. (a) Tokamak poloidal cross-section with the location of the ion cyclotron resonance layers for thermal $\mathrm{D}$ and $\mathrm{H}$ ions for JET pulse \#91256 $\left(B_{0} \approx 2.9 \mathrm{~T}, I_{\mathrm{p}}=2 \mathrm{MA}\right.$, $f=25 \mathrm{MHz}$ ). The computed locations of the ion-ion hybrid layer in H-D plasmas are also shown for $X[\mathrm{D}]=15 \%$ and $X[\mathrm{D}]=30 \%$ ( $n_{\mathrm{tor}}=27$ and $X\left[{ }^{9} \mathrm{Be}\right]=0.5 \%$ assumed $)$. (b) The parallel fast-ion distributions for tangential NBI injectors used in \#91256, as computed by the ASCOT code.

Mixed H-D plasmas with different isotopic compositions can be used to realize the 3-ion D-( $\left.\mathrm{D}_{\mathrm{NBI}}\right)-\mathrm{H}$ scheme. Yet there is an upper boundary on the concentration of D ions for this heating scheme to be efficient, $X[\mathrm{D}]_{\max }$ that is determined by the selected NBI and ICRF settings. First, we note the expression for the Doppler-shift of fast ions

$$
\delta R_{\text {fast }}=n_{\text {torV } \| \text {,fast }} / \omega,
$$

where we assumed $k_{\|}=n_{\text {tor }} / R$. A simple estimate for the maximum parallel velocities in the slowing-down distribution of the injected D-NBI ions yields $\mathrm{v}_{\| \text {, fast }}^{(\max )} \approx 2 \times 10^{6} \mathrm{~m} / \mathrm{s}$ $\left(E_{\mathrm{NBI}}=100 \mathrm{keV}, \lambda \approx 0.62\right)$, which is consistent with the results of the ASCOT modeling [19] shown in Fig. 2(b). Taking into account that ICRF power was coupled at $f=25 \mathrm{MHz}$ using dipole antenna phasing with a characteristic $n_{\text {tor }} \approx 27$, Eq. (3) implies that the fastest D-NBI ions have a Doppler-shift $\delta R_{\text {fast }}^{(\max )} \approx 0.35-0.4 \mathrm{~m}$. In mixed H-D plasmas, the latter quantity defines $X[\mathrm{D}]_{\max }$ as follows 


$$
X[\mathrm{D}]_{\max } \approx p /(1-p), p=\delta R_{\text {fast }}^{(\max )} / R_{0} .
$$

This gives $X[\mathrm{D}]_{\max } \approx 15 \%$ for the experimental conditions adapted in \#91256.

Figure 2(a) also shows the computed location of the ion-ion hybrid pair at higher concentration of D ions, $X[\mathrm{D}]=30 \%$. In this case $R_{\mathrm{IIH}} \approx 3.3-3.4 \mathrm{~m}$, meaning that fast ions with a Doppler shift of about $0.7-0.8 \mathrm{~m}$ are required to resonate at the IIH layer. For the ICRF settings used in \#91256, this corresponds to the parallel fast-ion velocities $\mathrm{v}_{\|} \approx 4.1-4.7 \times 10^{6} \mathrm{~m} / \mathrm{s}$. Fast ions with such large $\mathrm{v}_{\|}$are virtually absent in the slowing-down distribution of injected NBI ions (Fig. 2(b)) and, thus, the 3-ion scheme is not efficient under these conditions. In the absence of resonant ions fulfilling Eq. (1) at the ion-ion hybrid layer, the fast wave undergoes the transformation into the short wavelength modes and the traditional mode conversion heating is recovered. It is still possible to set up the 3-ion scheme in mixed H-D plasmas at larger D concentrations, but this requires higher NBI injection energies and/or ICRF operation with larger parallel wavenumbers, $k \|$.

A first proof for core RF power deposition in \#91256 is the response of the central electron temperature to the applied ICRF power. We complement this result (Fig. 1(b)) with $T_{\mathrm{e}}$-profiles corresponding to different heating phases of the pulse. Figure 3(a) shows that the NBI-only heating phase (with a rather broad deposition of NBI heating power) is characterized by a fairly flat $T_{\mathrm{e}}$-profile. Higher values for $T_{\mathrm{e}}(0)$ and more peaked $T_{\mathrm{e}}$-profiles are observed during the combined ICRF + NBI heating phases. The increase of the central electron temperature per MW of injected auxiliary heating power is significantly higher for the heating phase with combined ICRF + NBI heating, $\Delta T_{\mathrm{e}}(0) / \Delta P_{\mathrm{ICRF}} \approx 0.65 \mathrm{keV} / \mathrm{MW}$, as compared to the NBI-only phase, $\Delta T_{\mathrm{e}}(0) / \Delta P_{\mathrm{NBI}} \approx 0.2 \mathrm{keV} / \mathrm{MW}$.

We note that the $T_{\mathrm{e}}$-analysis alone does not allow to conclude whether the 3-ion ICRF scheme is the dominant channel of RF power deposition in \#91256. Indeed, core electron heating can be equally achieved with on-axis mode conversion heating in JET, e.g. [10]. However, as discussed in Refs. [10,20], short-wavelength modes, such as ion Bernstein waves, are characterized by much larger perpendicular wavenumbers than fast waves $\left(k_{\perp, \mathrm{IBW}} \gg k_{\perp, \mathrm{FW}}\right)$, and, as a result, interaction between ions and mode converted waves cannot lead to the generation of fast-ion populations. Thus, the presence or absence of fast D ions with energies significantly above NBI injection energies demonstrates whether the 3-ion scheme or electron mode conversion ICRF heating dominates.
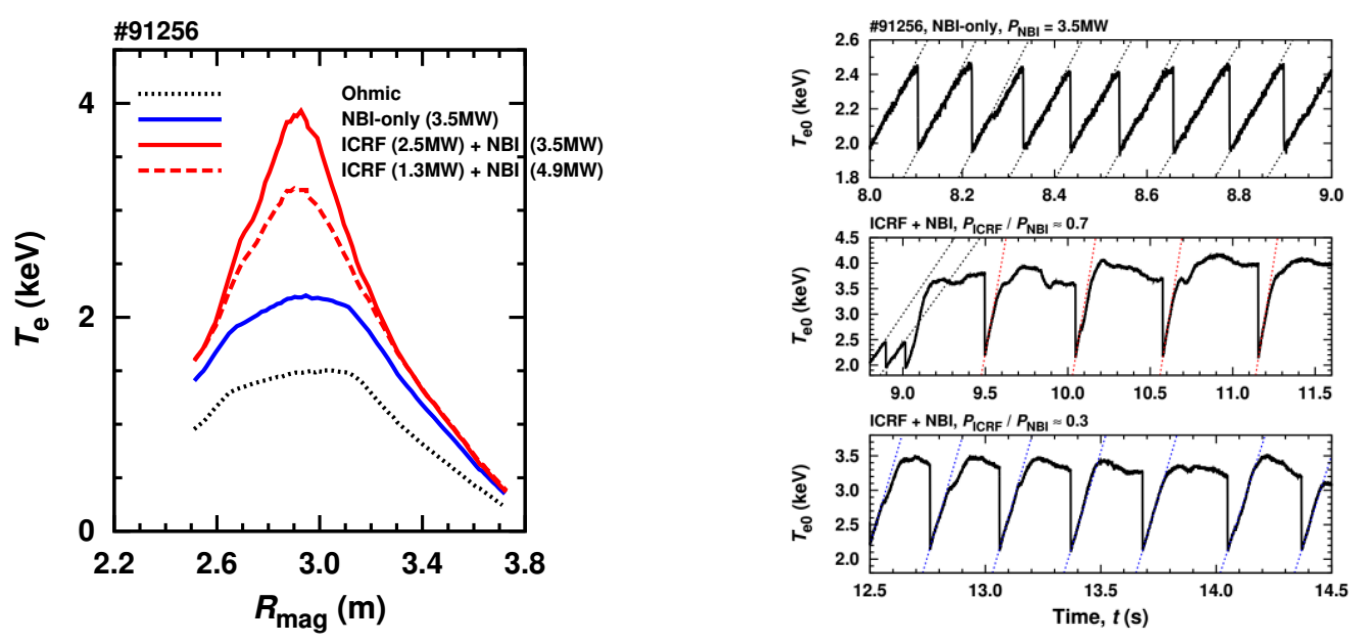

Figure 3. (a) $T_{\mathrm{e}}$-profiles (averaged over $0.5 \mathrm{~s}$ ) and (b) post-sawtooth recovery rate of $T_{\mathrm{e}}(0)$ in different heating phases of JET pulse \#91256. Centrally peaked electron temperature profiles are achieved when ICRF power is added on top of NBI power. 
A first indication for the presence of fast ions with energies above $E_{\mathrm{NBI}}$ are the long sawtooth periods in \#91256 during the phases with ICRF. While the $3.5 \mathrm{MW}$ NBI-only phase is characterized by an averaged sawtooth period $T_{\text {saw }} \approx 110 \mathrm{~ms}$, it increases to $T_{\text {saw }} \approx 550 \mathrm{~ms}$ in the combined ICRF +NBI phase with $2.5 \mathrm{MW}$ of ICRF power (Fig. 3(b)). Efficient generation of high-energy D ions with ICRF is further confirmed by the observation of a strongly increased neutron rate (by a factor of 10-15), as shown in Fig. 1(d), and an increased number of gamma-ray counts with $E_{\gamma}=3.37 \mathrm{MeV}$ (Fig. 1(e)). The observed gammas originate from one of the branches of the nuclear reactions between fast $\mathrm{D}$ ions and intrinsic ${ }^{9} \mathrm{Be}$ impurities. Given the fact that this nuclear reaction has an effective energy threshold $E_{\mathrm{D}}>0.5 \mathrm{MeV}$ [21], we can conclude that an acceleration of fast D ions with ICRF waves must have taken place. In turn, this implies that the mode conversion heating is not effective for the adapted scenario and the energetic D ions are generated as a result of their interaction with fast waves. The determining role of ICRF for accelerating D ions to high energies is further illustrated in the next phase of \#91256 with $P_{\mathrm{ICRF}} / P_{\mathrm{NBI}} \approx 0.3$ $\left(P_{\mathrm{ICRF}} \approx 1.3 \mathrm{MW}, P_{\mathrm{NBI}} \approx 4.9 \mathrm{MW}\right)$. Although the total auxiliary heating power (ICRF $+\mathrm{NBI}$ ) was approximately the same as in the earlier phase with $P_{\mathrm{ICRF}} / P_{\mathrm{NBI}} \approx 0.7$, we note shorter sawtooth periods ( $T_{\text {saw }} \approx 300 \mathrm{~ms}$ ) and a reduced number of gamma-ray counts and neutron rate.

In the short phase following a sawtooth crash, the central electron temperature recovers after a crash and linearly increases in time. We take the post-sawtooth $T_{\mathrm{e}}(0)$ recovery rate, $\mathrm{d} T_{\mathrm{e}}(0) / \mathrm{dt}$, as a figure-of-merit to characterize electron heating power densities in the plasma core [22]. As can be seen in Fig. 3(b), this quantity is significantly higher during the combined ICRF + NBI heating phases $\left(\sim 20 \mathrm{keV} / \mathrm{s}\right.$ at $P_{\mathrm{ICRF}} \approx 2.5 \mathrm{MW}$ and $\sim 11 \mathrm{keV} / \mathrm{s}$ at $\left.P_{\text {ICRF }} \approx 1.3 \mathrm{MW}\right)$, as compared to the NBI-only phase $(\sim 5 \mathrm{keV} / \mathrm{s})$. In the next section of the paper, we will show the detailed fast-ion measurements for \#91256, confirming that the fast $\mathrm{D}$ tail extends to the energies of $\sim 1.5 \mathrm{MeV}$. Note that the achieved fast-ion energies are significantly higher than the critical energy $E_{\text {crit }}(\mathrm{D}) \approx 28 T_{\mathrm{e}}$, and collisional electron heating from fast ions dominates. Thus, the comparison with $\mathrm{d} T_{\mathrm{e}}(0) / \mathrm{dt}$ rates also confirms that ICRF is the dominant source of electron heating in the plasma core.

From the observations of the neutron rate, sawtooth stabilization and gamma-ray counts, shown in Fig. 1, it is clear that the ratio $P_{\mathrm{ICRF}} / P_{\mathrm{NBI}}$ can be used as an actuator to control the achieved fast-ion energies with this ICRF +NBI synergetic scheme. This is further supported by PION modeling of \#91256, as reported in [16]. It is important to emphasize that the optimal values for $P_{\mathrm{ICRF}} / P_{\mathrm{NBI}}$ are different for the D-D, D- ${ }^{3} \mathrm{He}$ and D-T fusion reactions and are determined by the energy dependencies of the fusion cross-sections. While higher values of $P_{\mathrm{ICRF}} / P_{\mathrm{NBI}}$ are beneficial to generate $\mathrm{MeV}$-range $\mathrm{D}$ ions and maximize the $\mathrm{D}-\mathrm{D}$ neutron rate, the optimal energies of fast $\mathrm{D}$ and $\mathrm{T}$ ions are in the range $\sim 150-200 \mathrm{keV}$ for the D-T fusion reaction. The possibility to tailor the fast-ion energies is one of the advantages of this novel heating scheme.

The NBI system at JET consists of two neutral beam injector boxes, each equipped with eight Positive Ion Neutral Injectors (PINIs). Four PINIs in each box (\#1, \#2, \#7, \#8) are grouped into a 'tangential' bank and four in a 'normal' bank (\#3, \#4, \#5, \#6) [23]. Note that neither of the injectors are actually fully normal nor fully tangential to the magnetic field: fast ions injected by 'normal' PINIs are characterized by lower parallel velocities (characteristic $\lambda \approx 0.44$ ). Furthermore, depending on the trajectory of NBI injectors through the plasma, JET PINIs are additionally sub-classified as 'on-axis' (\#1, \#2, \#7, \#8) and 'offaxis' (\#3, \#4, \#5, \#6). 
The choice of NBI PINIs, injecting the fast particles that interact with the ICRF waves is another important actuator to control the fast-ion energies with the 3-ion ICRF + NBI scheme. This is clearly illustrated in JET pulse \#91255, see Fig. 4(a). The central $T_{\mathrm{e}}(0)$ and fast-D energies during the combined ICRF + NBI phase with off-axis normal PINIs (\#3, \#4) are clearly lower than for the two other ICRF + NBI phases in \#91255 $(\mathrm{D} /(\mathrm{H}+\mathrm{D}) \approx 0.09-0.11)$. This is corroborated by the changes in the sawtooth period. Note that the NBI acceleration voltage $E_{\mathrm{NBI}}=80 \mathrm{keV}$ in $\# 91255$ was lower compared to $E_{\mathrm{NBI}}=100 \mathrm{keV}$ in \#91256.

It is instructive to compare the neutron decay phases in \#91255. We start this analysis for the NBI-only phase $(t=8.0-8.6 \mathrm{~s})$, in which the fast-ion energies are limited by NBI acceleration voltage. After switching off the NBI system, the neutron rate exponentially decreases with a decay time $\tau_{\mathrm{n}} \approx 13 \mathrm{~ms}$. The analysis of the corresponding phase after the combined ICRF + NBI heating $(t=12.8-13.4 \mathrm{~s})$ reveals a much longer $\tau_{\mathrm{n}} \approx 300 \mathrm{~ms}$ (reduced to $\tau_{\mathrm{n}} \approx 100 \mathrm{~ms}$ after the sawtooth crash at $t \approx 13.58 \mathrm{~s}$ ). The increased neutron decay time should be partly attributed to higher electron temperatures reached during the combined ICRF + NBI phase. Since $\tau_{\mathrm{S}} \propto T_{\mathrm{e}}^{3 / 2}$, the effect of a higher $T_{\mathrm{e}}$ on the fast beam ion population should provide an increase of the slowing-down time of the fast $\mathrm{D}$ ions by a factor of 3 , should the fast-D energies be limited by the NBI acceleration voltage. But, as the observed increase in the neutron decay time is much larger $(300 \mathrm{~ms}$ vs. $13 \mathrm{~ms})$, this confirms an acceleration of the injected NBI ions to higher energies by the ICRF waves. A detailed analysis of the evolution of the fast-D population during the neutron decay phase is outside the scope of this paper and will be reported elsewhere.

We also note that for the selected mixed H-D plasmas, applying ICRF power alone is not effective either in generating a population of high-energy D ions in the plasma core. Figure 4(a) shows that the averaged sawtooth period during the ICRF-only phase $(t=14.0-15.0 \mathrm{~s})$ is as low as $50 \mathrm{~ms}$, and is even shorter than the corresponding value during the NBI-only phase ( $\left.T_{\text {saw }} \approx 80 \mathrm{~ms}\right)$.
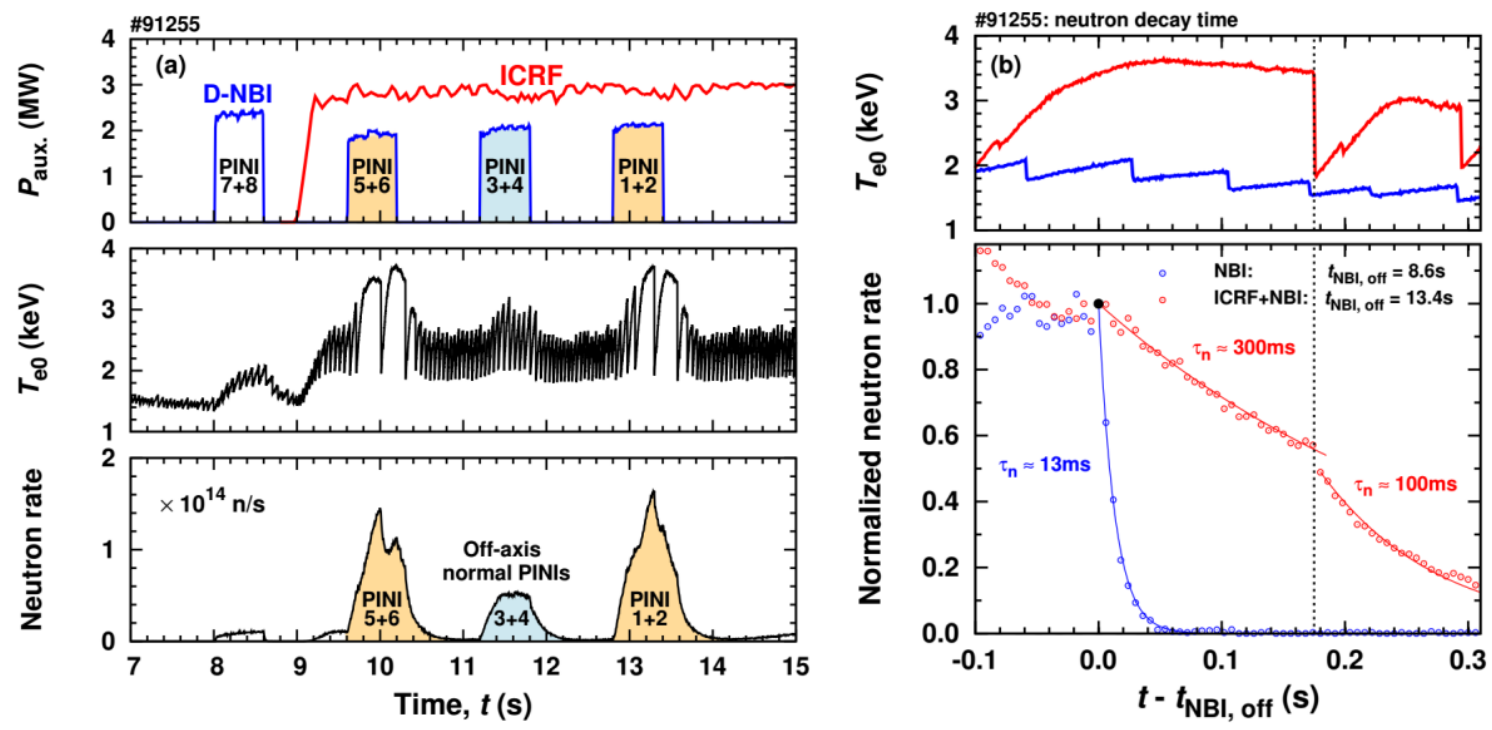

Figure 4. (a) The 3-ion D-( $\left.\mathrm{D}_{\mathrm{NBI}}\right)-\mathrm{H}$ heating scheme, overview of JET pulse \#91255 $\left(E_{\mathrm{NBI}}=80 \mathrm{keV}\right)$. (b) Exponential decay of the neutron rate starts after NBI system is switched off: NBI-only phase: $t_{\mathrm{NBI}}$, off $=8.6 \mathrm{~s}, \mathrm{ICRF}+\mathrm{NBI}$ phase (injectors \#1 and \#2): $t_{\mathrm{NBI} \text {, off }}=13.4 \mathrm{~s}$. Much longer neutron decay times are observed after the ICRF $+\mathrm{NBI}$ heating phase. 


\section{Summary of fast-ion observations in JET pulse \#91256}

JET is equipped with an enhanced suite of diagnostics for measuring both confined and lost energetic ions $[24,25]$. This section summarizes fast-ion measurements in the combined ICRF + NBI heating phase of JET pulse \#91256 $(t=9.0-11.6 \mathrm{~s})$, in which a strong increase in the neutron rate was observed when adding $P_{\text {ICRF }}=2.5 \mathrm{MW}$ of ICRF power (see Fig. $1(\mathrm{~d})$ ). In what follows, we show the consistent physical picture that emerged from a range of fastion measurements. These allowed us to quantify the energies of the resonant D-NBI ions accelerated by the ICRF waves and validate the physics aspects behind this novel heating scheme. Furthermore, analysis of fast-ion measurements provides information on the spatial localization of the confined energetic D ions in the plasma, confirming their very close localization at the ion-ion hybrid layer as a result of the interaction with ICRF waves. Figure 5 shows the lines-of-sight of different JET fast-ion diagnostics discussed in this section.

In mixed H-D plasmas, most of the neutrons are generated from one of the two channels of the D-D fusion reaction

$$
\mathrm{D}+\mathrm{D} \rightarrow{ }^{3} \mathrm{He}(0.82 \mathrm{MeV})+n(2.45 \mathrm{MeV}) .
$$

The neutron energy spectrum carries information on the distribution of the thermal and supra-thermal D ions in the plasma. In particular, we note that the neutron spectrum extends to energies well above $2.45 \mathrm{MeV}$ if a significant population of high-energy $\mathrm{D}$ ions is present in the plasma. As shown in [26-28], the analysis of the time-of-flight spectrometer TOFOR data has been applied for the interpretation of fast-ion experiments at JET and validating modeling results for the scenarios with $2^{\text {nd }}$ and $3^{\text {rd }}$ harmonic D ICRF heating.

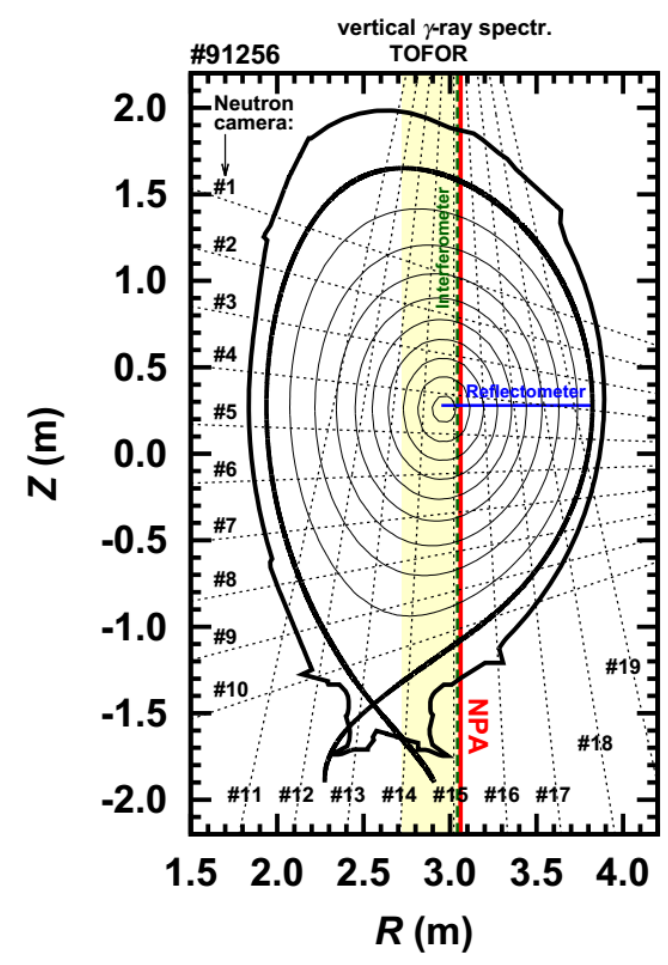

Figure 5. The lines-of-sight of different diagnostics applied for the interpretation of fast-ion measurements in JET pulse \#91256: the neutron camera (channels \#1-19, black dotted lines), the high-energy NPA (red vertical line), TOFOR and the vertical $\gamma$-ray spectrometer (yellow shaded area), the reflectometer (blue solid line) and the interferometer (green dashed line). 

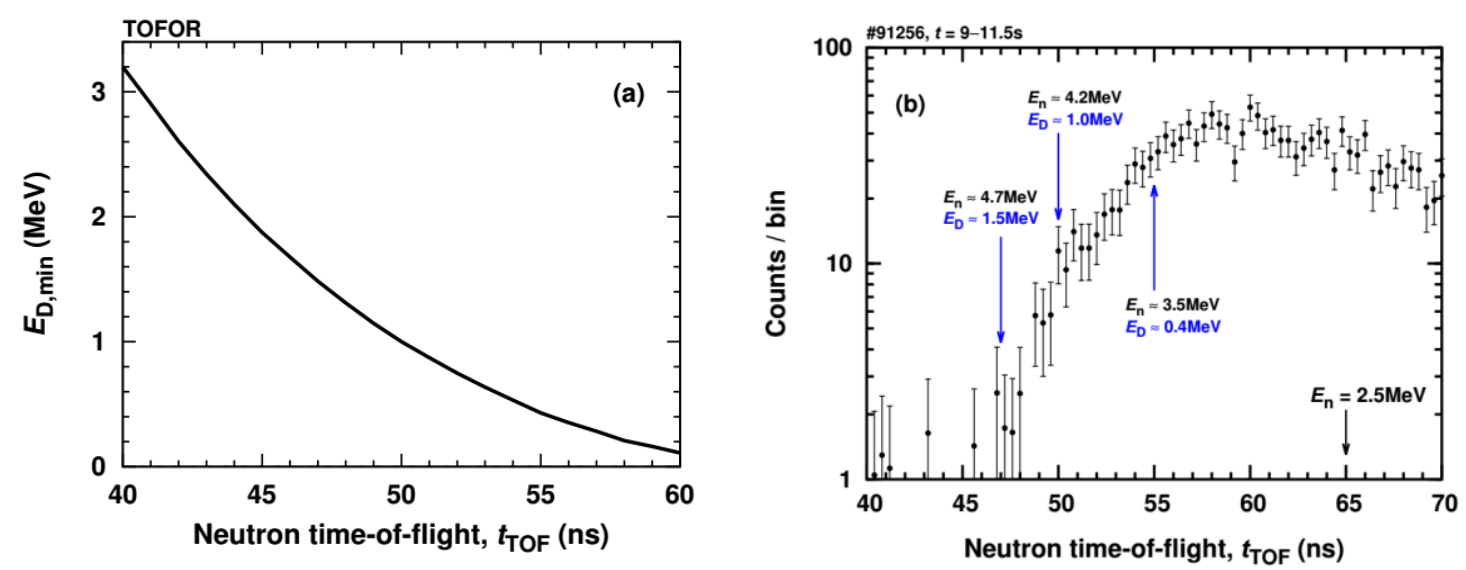

Figure 6. (a) Relation between the minimum energy of $D$ ions required for a given neutron time-of-flight in TOFOR (Fig. 3(b) in [29]). (b) TOFOR neutron energy spectrum for JET pulse \#91256, $t=9-11.5 \mathrm{~s}\left(P_{\mathrm{ICRF}} / P_{\mathrm{NBI}} \approx 0.7, P_{\mathrm{ICRF}} \approx 2.5 \mathrm{MW}, P_{\mathrm{NBI}} \approx 3.5 \mathrm{MW}\right)$.

TOFOR measures the time difference of detected neutrons between two sets of scintillators, and, as a first-order approximation, the mean TOFOR time-of-flight, $t_{\mathrm{TOF}}$ and the neutron energy are related as [27]

$$
E_{\mathrm{n}} \approx 2.9 \mathrm{MeV} \times\left(60 \mathrm{~ns} / t_{\mathrm{TOF}}\right)^{2} .
$$

Thus, shorter times-of-flight correspond to higher neutron energies, and higher neutron energies are a sign of more energetic $\mathrm{D}$ ions in the plasma. Based on the knowledge of the response function of TOFOR and from the kinematics of the D-D fusion reactions, a relation between a given time-of-flight and a minimum $\mathrm{D}$ energy corresponding to a given $t_{\text {TOF }}$ has been derived [29], see Fig. 6(a).

Figure 6(b) shows the TOFOR data for the combined ICRF and NBI heating phase of \#91256 with $P_{\mathrm{ICRF}} \approx 2.5 \mathrm{MW}$ and $P_{\mathrm{NBI}} \approx 3.5 \mathrm{MW}$, in which a strong enhancement of the neutron rate was observed (cf. Fig. 1(d)). The TOFOR spectrum extends to times-of-flight down to $t_{\mathrm{TOF}} \approx 47-50 \mathrm{~ns}$, corresponding to neutron energies $E_{\mathrm{n}} \approx 4.2-4.7 \mathrm{MeV}$. The observation of such high-energy neutrons is direct evidence for the presence of energetic D ions in the plasma with energies $E_{\mathrm{D}} \approx 1.0-1.5 \mathrm{MeV}$. This is further illustrated in Fig. 7, showing the sensitivity in velocity space for two TOFOR times-of-flight [30-32]. White regions correspond to domains in the velocity space where the diagnostic is not sensitive, and darker colored regions to increased TOFOR sensitivity. The dashed lines represent deuterium energies of $1 \mathrm{MeV}, 2 \mathrm{MeV}$ and $3 \mathrm{MeV}$, and the dotted lines show $0.2 \mathrm{MeV}$ steps. The clear signal at $t_{\mathrm{TOF}}=47 \mathrm{~ns}$ in TOFOR (cf. Fig. 6(b)) suggests the presence of deuterium ions with energies of at least $\sim 1.3 \mathrm{MeV}$. The absence of a significant TOFOR signal at $t_{\mathrm{TOF}}=46 \mathrm{~ns}$ suggests that very few ions reach energies larger than $\sim 1.5 \mathrm{MeV}$. We note that in the follow-up heating phase of \#91256 with lower ICRF power $\left(P_{\mathrm{ICRF}} \approx 1.3 \mathrm{MW}\right.$, $\left.P_{\mathrm{NBI}} \approx 4.9 \mathrm{MW}\right)$, the TOFOR spectrum extends only to $t_{\mathrm{TOF}} \approx 53-55 \mathrm{~ns}\left(E_{\mathrm{D}} \approx 0.5 \mathrm{MeV}\right)$. This result is consistent with the earlier discussion presented in section 2 that the developed heating scheme allows also to generate moderately energetic ions, e.g., by decreasing the ratio $P_{\mathrm{ICRF}} / P_{\mathrm{NBI}}$. 

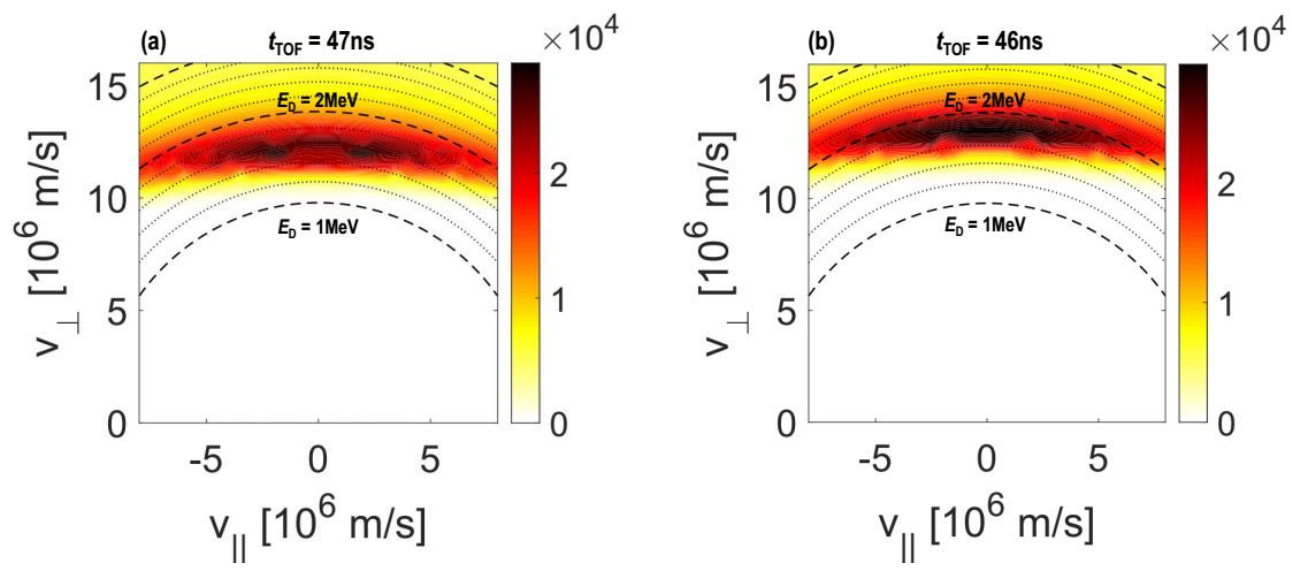

Figure 7. TOFOR weight functions for: (a) $t_{\mathrm{TOF}}=47 \mathrm{~ns}$ and (b) $t_{\mathrm{TOF}}=46 \mathrm{~ns}$.

The presence of high-energy D ions is also confirmed by measurements of the neutral particle analyzer (NPA). The high-energy NPA at JET has a vertical line-of-sight that passes through the core region of the plasma $\left(R_{\text {los }} \approx 3.06 \mathrm{~m}\right.$, cf. Fig. 5$)$. In pulse \#91256, fast D ions were detected by the NPA system in the energy channels ranging from $287 \mathrm{keV}$ to $952 \mathrm{keV}$. Figure 8(a) shows the temporal evolution of the neutral D flux at $E_{\mathrm{D}}=455 \mathrm{keV}$. As expected, NPA measurements of energetic D ions correlate with the observed enhancement of the neutron rate. The measured integrated neutral flux as a function of the deuterium energy for the combined ICRF + NBI phase with $P_{\mathrm{ICRF}}=2.5 \mathrm{MW}$ is shown in Fig. 8(b). It is clear that NPA measurements also confirm that the deuterium tail extends to the energies of $\sim 1 \mathrm{MeV}$.

The so-called synthetic NPA diagnostic [33] was applied for inferring the properties of a fast D distribution in JET pulse \#91256. In this analysis the fast-ion population was assumed to be a Maxwellian, characterized by an effective temperature as a free parameter. The two dotted lines in Fig. 8(b) show the energy-dependence of the predicted neutral flux for $T_{\text {eff }}=100 \mathrm{keV}$ and $T_{\text {eff }}=300 \mathrm{keV}$. The assumed $T_{\text {eff-values result in an under- and }}$ overestimation of the fast $\mathrm{D}$ population in the high-energy part of the distribution $\left(E_{\mathrm{D}}>600 \mathrm{keV}\right)$, respectively. The NPA synthetic modeling results and NPA measurements agree rather well for $T_{\text {eff }}=180 \mathrm{keV}$ (red solid line in Fig. 8(b)). Note that the inferred value is close to the effective D ion tail temperature predicted by PION and TRANSP simulations $[16,34]$.
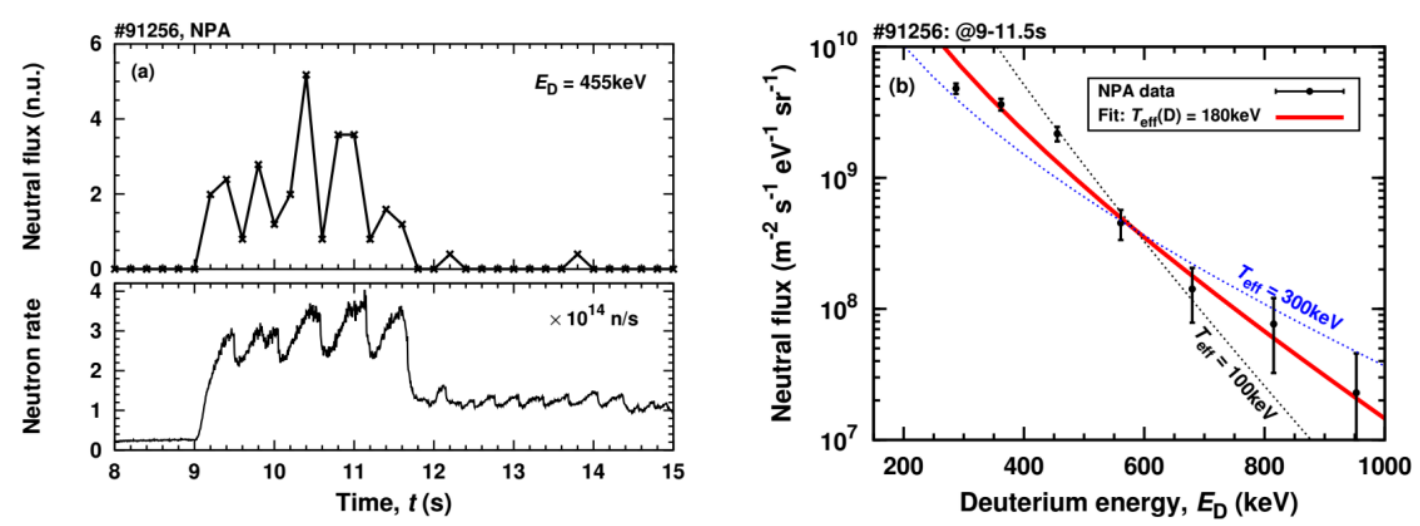

Figure 8. NPA measurements for JET pulse \#91256: (a) Temporal evolution of the measured neutral flux at $E_{\mathrm{D}}=455 \mathrm{keV}$. (b) The energy dependence of the integrated neutral flux during the combined ICRF $+\mathrm{NBI}$ phase with $P_{\mathrm{ICRF}}=2.5 \mathrm{MW}$ and $P_{\mathrm{NBI}}=3.5 \mathrm{MW}$. Synthetic NPA diagnostic fits for $T_{\mathrm{eff}}=100 \mathrm{keV}, 180 \mathrm{keV}$ and $300 \mathrm{keV}$ are also shown. 
Another independent confirmation for the presence of high-energy D ions generated with ICRF is provided by gamma-ray emission spectroscopy [35]. As a result of nuclear reactions between fast $\mathrm{D}$ ions and intrinsic ${ }^{9} \mathrm{Be}$ impurities, heavy nuclei in an excited state, ${ }^{10} \mathrm{Be}^{*}$ and ${ }^{10} \mathrm{~B}^{*}$, are produced [28]. When a heavy nucleus de-excites, this results in the emission of gamma-ray lines at discrete energies determined by each specific nuclear reaction, allowing an unambiguous identification of the fast-ion species. Lines at energies 2.87 MeV, $3.37 \mathrm{MeV}$ and $3.59 \mathrm{MeV}$ are the strongest peaks in the recorded gamma-ray spectrum in pulse \#91256, confirming that the observed gamma-ray emission is originating due to the presence of fast $\mathrm{D}$ ions in the plasma. Furthermore, as $\mathrm{D}+{ }^{9} \mathrm{Be}$ nuclear reactions have an effective energy threshold $E_{\mathrm{D}} \approx 0.5 \mathrm{MeV}$ for their detection at JET [21], the temporal evolution of the number of $\gamma$-counts reflects how the number of high-energy $\mathrm{D}$ ions changes with varying heating conditions in \#91256. The evolution of the $\gamma$-counts at $E_{\gamma}=3.37 \mathrm{MeV}$ has been earlier shown in Fig. 1(d). Note that its dynamics is very similar to that of the NPA counts (Fig. 9(a)). Fast D ions with energies larger than $0.5 \mathrm{MeV}$ are only present in the $\mathrm{ICRF}+\mathrm{NBI}$ heating phase of \#91256 with larger $P_{\mathrm{ICRF}} / P_{\mathrm{NBI}}$, highlighting the determining role of ICRF for accelerating the $\mathrm{D}$ ions to high energies.

The spatial distribution of fast $\mathrm{D}$ ions in the plasma was inferred from the analysis of neutron camera data [36]. This system at JET consists of 10 horizontal and 9 vertical channels. Figure 9 shows the neutron camera lines-of-sight and the neutron flux per channel, measured during the combined ICRF + NBI heating phase with $P_{\text {ICRF }}=2.5 \mathrm{MW}$ and $P_{\mathrm{NBI}}=3.5 \mathrm{MW}(t=9.8-10.0 \mathrm{~s})$. As follows from the figure, most of the neutrons were measured by the horizontal channels \#4 and \#5, and the vertical channels \#14 and \#15. This result proves that using this novel heating scheme RF power was deposited in the plasma core, giving rise to the strongly core-localized fast-ion population.
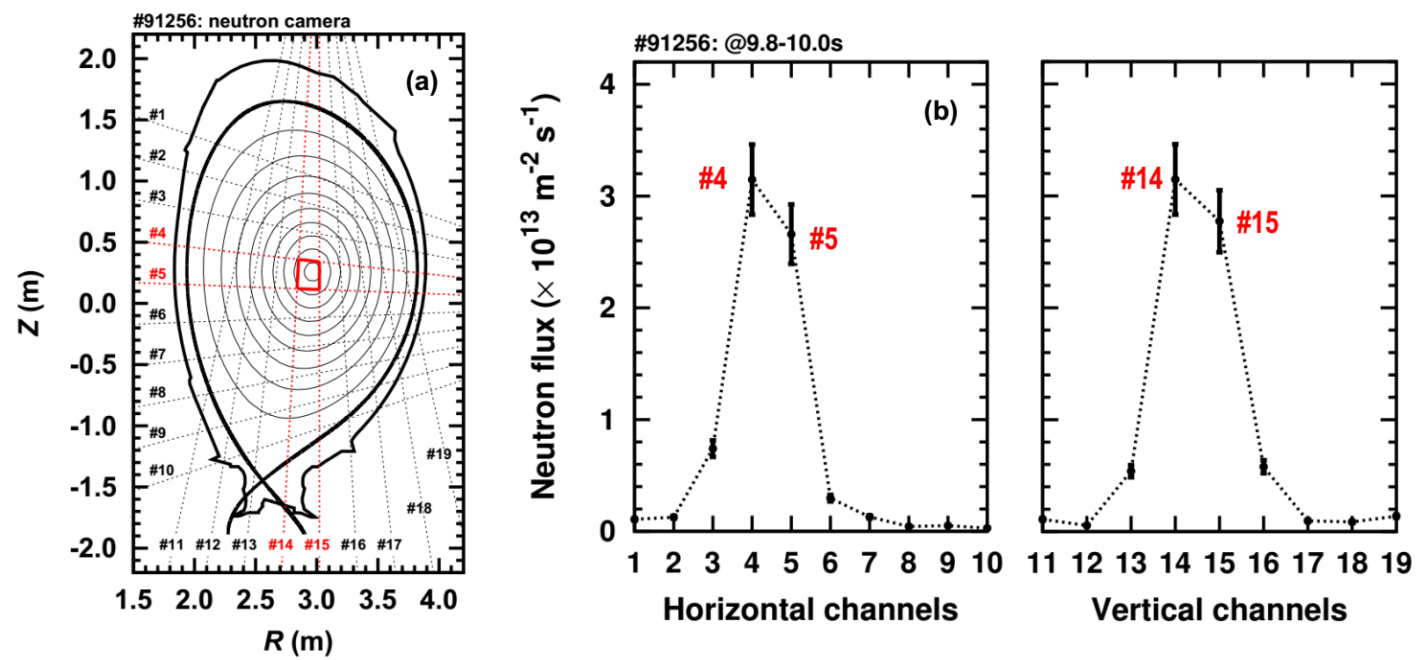

Figure 9. (a) Lines-of-sight of the neutron camera at JET. (b) Neutron flux for individual camera channels, measured during the ICRF + NBI heating phase with $P_{\text {ICRF }}=2.5 \mathrm{MW}$, $P_{\mathrm{NBI}}=3.5 \mathrm{MW}$ in pulse \#91256 $(t=9.8-10.0 \mathrm{~s})$. 
We recall that the strong localization of RF power deposition with the novel 3-ion ICRF + NBI scheme is achieved by making good use of the strong local enhancement of the left-hand RF electric field in the vicinity of the ion-ion hybrid layer. This is illustrated in Fig. 10(a), showing that the computed spatial profile of $\left|E_{+}\right|^{2}$ is enhanced in the plasma core in the vicinity of the IIH layer in H-D mixed plasmas. The computations were done with the 2D full-wave code TORIC [37,38], predicting that more than $80 \%$ of the incoming RF power is absorbed by fast D-NBI ions for the conditions of pulse \#91256. Figure 10 shows good overall agreement between the computed spatial profile of $\left|E_{+}\right|^{2}$ and the reconstructed neutron emission [39], using neutron camera data as input (Fig. 9(b)). This result highlights that the IIH layer indeed acts as a spatial filter because of the very efficient ion cyclotron wave-particle interaction that takes place in the close vicinity of this layer. Note that the developed 3-ion ICRF + NBI scheme features strong single-pass wave damping and this is particularly important for plasma heating with ICRF waves in fusion devices with a metallic wall.
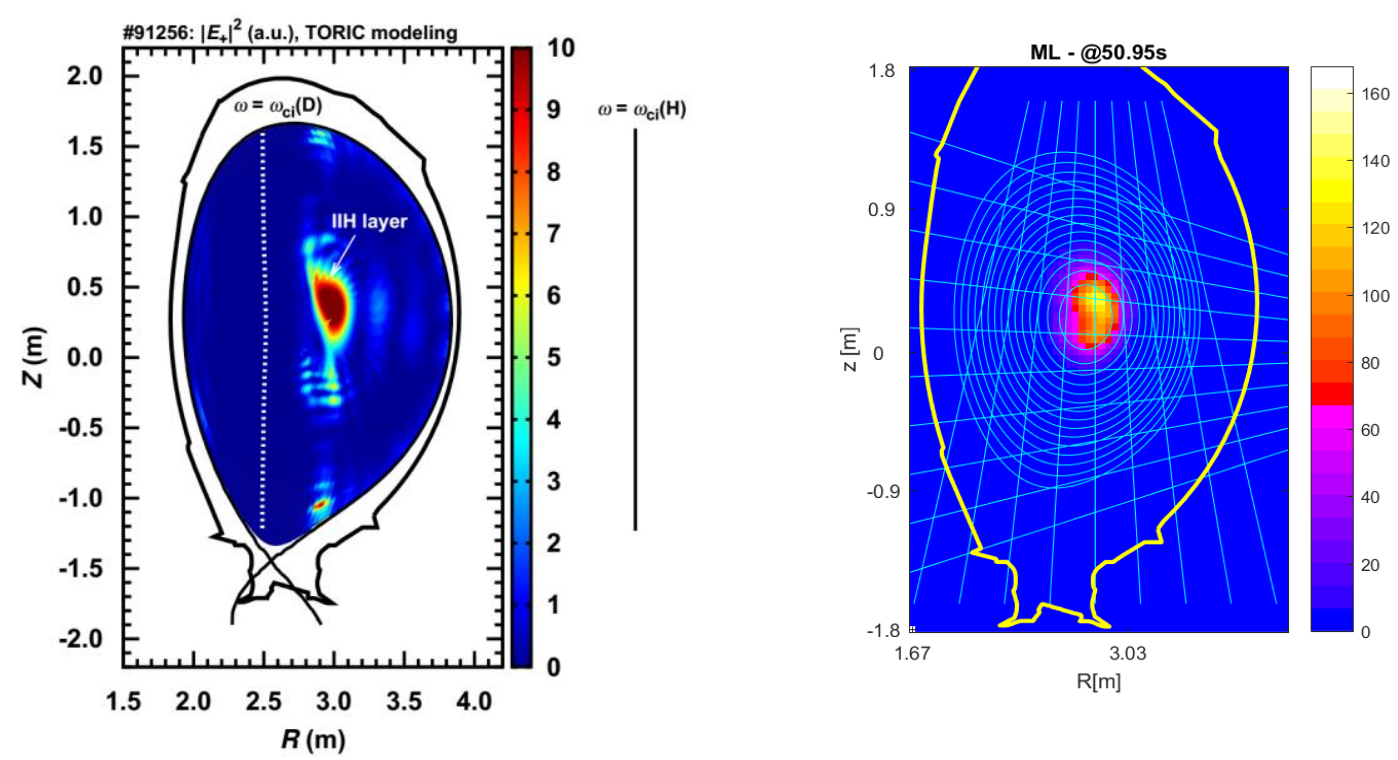

Figure 10. Illustration of the strong core localization of the RF power deposition and generation of energetic D ions in JET pulse \#91256. (a) Spatial distribution of the square of the left-hand RF electric field (computed by the 2D full-wave code TORIC [37]) exhibits the strong enhancement of $\left|E_{+}\right|^{2}$ at the IIH layer in the plasma core. (b) The reconstructed neutron emission, using the neutron camera data (Fig. 8(b)).

An independent confirmation of the core localization of fast ions in JET pulse \#91256 was inferred from the analysis of MHD modes. Figures 11(a) and (b) show spectrograms from core interferometer and Mirnov coil signals, illustrating the presence of fast-ion driven MHD modes at frequencies $\sim 90-100 \mathrm{kHz}$ and $\sim 310-360 \mathrm{kHz}$ during the combined $\mathrm{ICRF}+\mathrm{NBI}$ phase with $P_{\mathrm{ICRF}}=2.5 \mathrm{MW}$ and $P_{\mathrm{NBI}}=3.5 \mathrm{MW}$. These MHD modes were neither observed during the NBI-only phase of the pulse nor during the combined $\mathrm{ICRF}+\mathrm{NBI}$ phase with lower $P_{\mathrm{ICRF}} / P_{\mathrm{NBI}}\left(P_{\mathrm{ICRF}}=1.3 \mathrm{MW}\right.$ and $\left.P_{\mathrm{NBI}}=4.9 \mathrm{MW}\right)$. The radial localization of the observed MHD modes was determined using the X-mode correlation reflectometer [40]. The system consist of two independent fast-hopping reflectometers using the same waveguides for the mid-plane horizontal line-of-sight (blue solid line in Fig. 5) [41]. The reflectometer was set to scan radially from the plasma edge to the plasma core with a scan cycle time of about $0.45 \mathrm{~s}$. As follows from Figs. 11(c) and (d), the observed MHD 

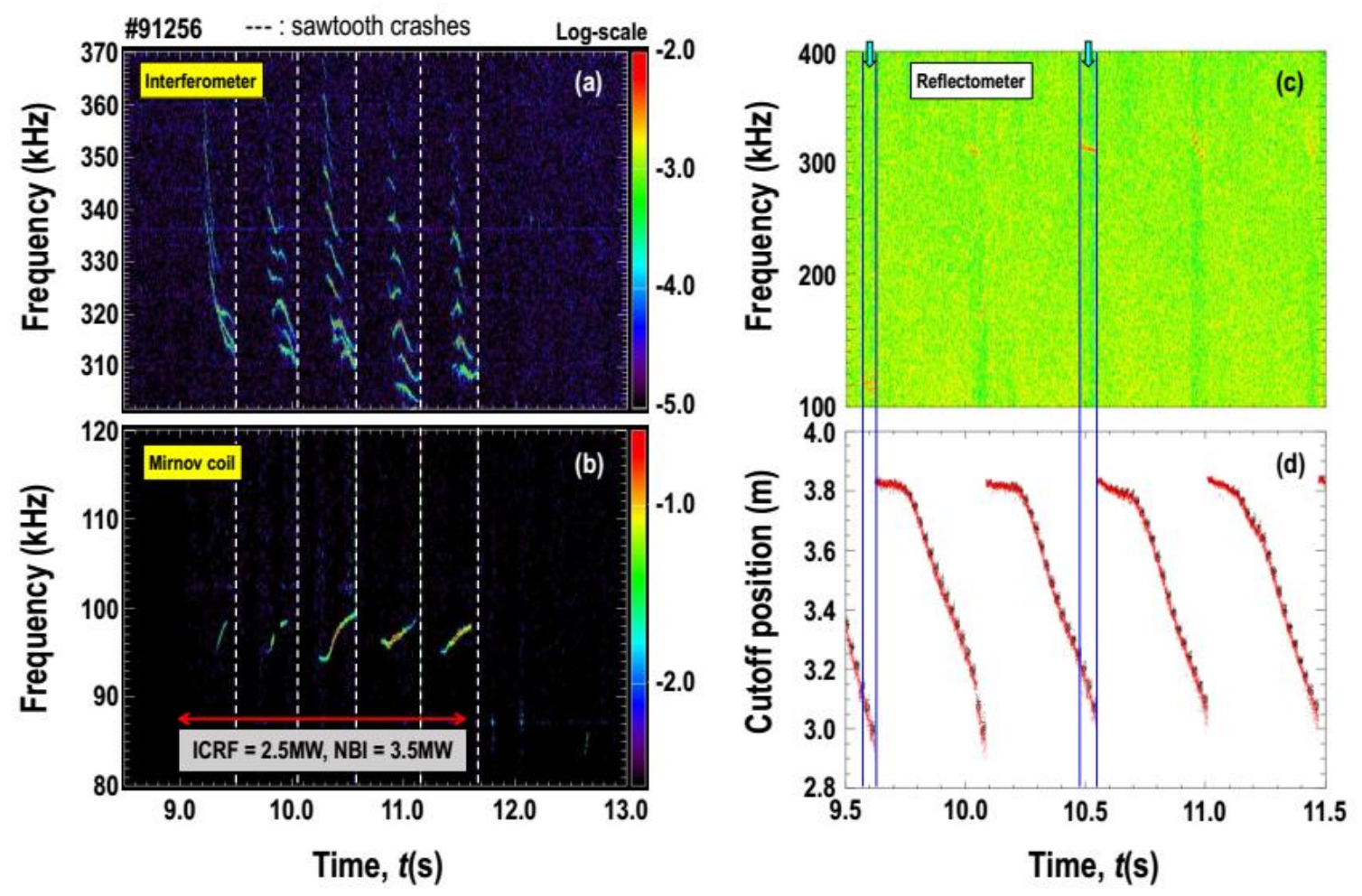

Figure 11. Spectrograms from the core interferometer (a), Mirnov coil (b) and radial correlation reflectometer signals, showing MHD modes in the ICRF + NBI heating phase of JET pulse \#91256. The observed MHD modes are core-localized as follows from the correlation reflectometer signal analysis: (c) and (d).

modes are localized in the plasma core, $R<3.2 \mathrm{~m}$. The radial localization of the MHD modes is consistent with the computed location of the IIH layer in the plasma and the reconstructed neutron emission (Fig. 10). Note that $R \approx 3.2 \mathrm{~m}$ corresponds to the location of the $q=1$ surface, as inferred from the analysis of the sawtooth inversion radius.

\section{Outlook of possible applications and future studies}

The application of the novel 3-ion schemes is not limited to mixed H-D plasmas and can be easily extended for its use in different type of plasma mixtures, e.g., D- ${ }^{3} \mathrm{He}$ and D-T. As shown in the paper, the scheme allows to vary the fast-ion energy from moderately high to very high, depending on the experimental conditions. Generating a large number of $\mathrm{MeV}$ range ions is of interest not only for studying the physics of fast ions and MHD modes, but also to mimic the heating conditions typical for D-T plasmas in ITER and a fusion reactor. Indeed, these plasmas are characterized by strong electron heating with fusion-born alpha particles as the main heating source. As follows from recent gyrokinetic analysis, alpha particles in ITER can significantly stabilize ITG turbulence and reduce heat transport and energy losses [42]. Thus, the 3-ion ICRF schemes at JET, tuned for maximizing the generation of fast ions in the plasma core and characterized by strong fast-ion electron heating, provide a test bed to elucidate the role of fast ions on microturbulence and clarify the impact of alpha particles in future ITER plasmas. 
In the context of this application, interesting observations were made in very recent JET experiments, in which the 3-ion ICRF scheme D-( $\left.\mathrm{D}_{\mathrm{NBI}}\right)-{ }^{3} \mathrm{He}$ was applied in L-mode D- ${ }^{3} \mathrm{He}$ mixed plasmas $\left(n\left({ }^{3} \mathrm{He}\right) / n_{\mathrm{e}} \approx 20-25 \%\right)$. As for pulse \#91256, the experimental conditions were chosen such that the ion-ion hybrid layer is located in the plasma core and to use injected fast D-NBI ions as a resonant species to absorb RF power in the vicinity of the IIH layer. Note that the high-efficiency of this heating scheme in $\mathrm{D}-{ }^{3} \mathrm{He}$ plasmas at $n\left({ }^{3} \mathrm{He}\right) / n_{\mathrm{e}} \approx 25 \%$ was earlier observed in JET with the carbon wall, discussed in detail in section 4 in Ref. [10]. Figure 12 shows an overview of the JET pulse \#94701 (3.7T/2.5MA), in which $6 \mathrm{MW}$ of ICRF was applied in combination with $8 \mathrm{MW}$ of NBI. The neutron rate was increased from $\sim 0.6 \times 10^{15} \mathrm{n} / \mathrm{s}$ in the NBI-only phase to $\sim 1.0 \times 10^{16} \mathrm{n} / \mathrm{s}$ in the combined ICRF + NBI phase of the pulse. For comparison, we also illustrate the plasma performance for an NBI-only pulse \#94704 (dominant ion heating in the core), which was performed at the same operational conditions $\left(B_{t} / I_{\mathrm{p}}, n_{\mathrm{e}}, n\left({ }^{3} \mathrm{He}\right) / n_{\mathrm{e}}\right.$, plasma shape, etc). The stronger $T_{\mathrm{e}}$ peaking and much higher $T_{\mathrm{e} 0}$ and neutron rate in pulse \#94701, compared to \#94704, are easily understood by the presence of high-energy $\mathrm{D}$ ions due to efficient central deposition of ICRF power. However, the comparison of the measured plasma stored energy, $T_{\mathrm{e}}$ and $T_{\mathrm{i}}$ profiles at the same total auxiliary heating power, $P_{\text {aux. }}=14 \mathrm{MW}$ and plasma density, $n_{\mathrm{e} 0} \approx 6 \times 10^{19} \mathrm{~m}^{-3}$, as shown in Figs. 12 (e), (f) and (g), hints at a significantly reduced transport in pulse \#94701, characterized by a large fraction of fast ions in the plasma core.
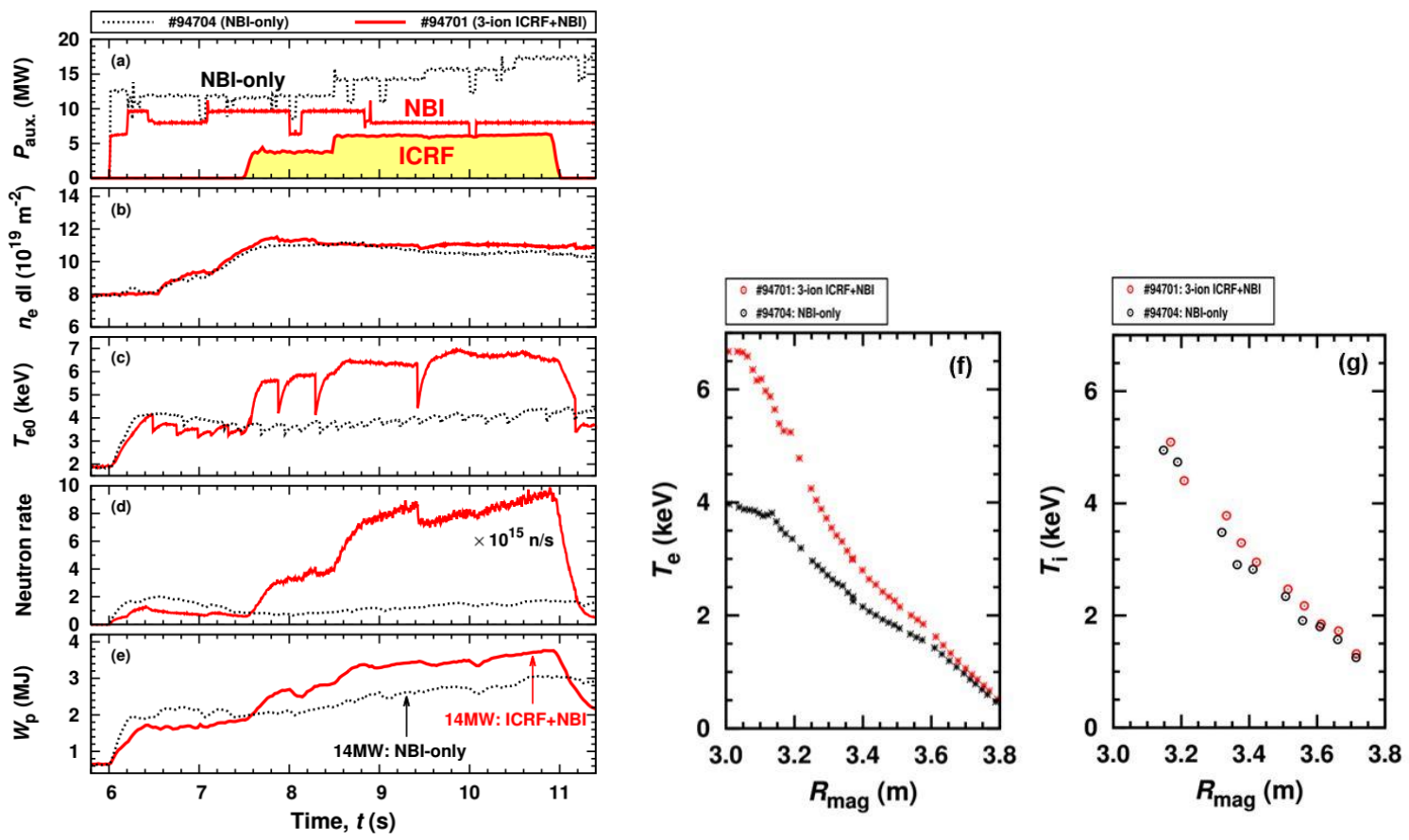

Figure 12. Overview of JET pulse \#94701, in which the 3 -ion ICRF scheme D-(D $\left.\mathrm{D}_{\mathrm{NBI}}\right){ }^{3} \mathrm{He}$ was applied for plasma heating and fast-ion generation in $\mathrm{D}^{3} \mathrm{He}$ mixed plasma (L-mode, $3.7 \mathrm{~T} / 2.5 \mathrm{MA}, n_{\mathrm{e} 0} \approx 6 \times 10^{19} \mathrm{~m}^{-3}, n\left({ }^{3} \mathrm{He}\right) / n_{\mathrm{e}} \approx 20-25 \%$, ICRF: $f=33 \mathrm{MHz}$, dipole phasing; NBI: $E_{\mathrm{NBI}}=95-100 \mathrm{keV}$, tangential injectors). The heating conditions in the plasma core mimic the conditions of ITER with dominant electron heating from fast ions. The dotted black lines give an overview of JET pulse \#94704 with NBI-only heating (dominant ion heating in the core) at the same operational conditions as in pulse \#94701. From top to bottom are displayed: (a) Auxiliary heating power; (b) Core line-integrated density; (c) Central electron temperature; (d) Neutron rate; (e) Plasma stored energy. (f) and (g) Comparison of $T_{\mathrm{e}}$ and $T_{\mathrm{i}}$ profiles for pulses \#94701 and \#94704 at the same total $P_{\text {aux. }}=14 \mathrm{MW}$ and plasma density, $n_{\mathrm{e} 0} \approx 6 \times 10^{19} \mathrm{~m}^{-3}$. 
The observation $T_{\mathrm{i}} / T_{\mathrm{e}} \approx 1$ in JET plasmas, featuring dominant core electron heating from fast ions, is very promising for ITER. The detailed transport analysis of pulse \#94701, aiming to understand better the transport in JET plasmas under the ITER-relevant heating conditions and identify possible fast-ion effects on microturbulence, is ongoing and the results will be reported elsewhere. We also note that the developed 3-ion scheme was used as a tool to generate a population of ${ }^{-}{ }^{3} \mathrm{He}$ fusion-born alpha particles $\left(E_{\alpha} \approx 3.6 \mathrm{MeV}\right)$ and to validate successfully updated JET diagnostics for alpha measurements prior to future D-T operations.

In D-T plasmas with an isotopic ratio varying between 20\%:80\% and 80\%:20\%, different resonant species can be used for exploiting the enhancement of $\left|E_{+}\right|^{2}$ at the IIH layers between the cyclotron resonances of thermal $\mathrm{D}$ and $\mathrm{T}$ ions and the realization of the 3-ion ICRF schemes. We note that RF power can be partly absorbed by several species simultaneously, including in particular ${ }^{9} \mathrm{Be}$ impurities, injected fast D-NBI or T-NBI ions, and fusion-born alpha particles. The split of RF power between these species depends on the experimental conditions such as the D:T ratio, ICRF and NBI settings (heating power, ICRF antenna phasing, NBI energies and injectors), plasma density and temperature, concentration of impurity species, the amount of fusion-born alphas, etc.

The 3-ion ICRF scheme with intrinsic ${ }^{9} \mathrm{Be}$ impurities to absorb RF power is a promising scenario for maximizing bulk ion heating and thermonuclear neutron rate in both JET and ITER D-T plasmas [43]. This scenario is relevant for heating D:T $=50 \%-50 \%$ plasmas with a particularly attractive feature that the intrinsic level of ${ }^{9} \mathrm{Be}$ impurities $(\sim 1 \%)$ is sufficient and no extra gas injection is required for the absorption of ICRF waves. The computations predict that these impurities, as a result of their relatively high atomic mass, transfer an even larger fraction of absorbed RF power to bulk D and T ions than ${ }^{3} \mathrm{He}$ minorities [15]. Dedicated experiments to validate bulk ion heating with ${ }^{9} \mathrm{Be}$ ICRF in $\mathrm{D}$ :T-equivalent $\mathrm{T}-{ }^{4} \mathrm{He}$ mixed plasmas are being prepared for the forthcoming tritium campaign at JET. Note that the ${ }^{9} \mathrm{Be}$ ICRF scheme is compatible with additional seeding of Ar and ${ }^{22} \mathrm{Ne}$ impurities, having very similar charge-to-mass ratio as ${ }^{9} \mathrm{Be}$.

Using T-NBI or D-NBI ions as a resonant species for the 3-ion ICRF scheme in D-T plasmas offers the possibility to accelerate fast T or D ions in JET to moderate energies of $\sim 100-200 \mathrm{keV}$. At these fast-ion energies the D-T reactivity is maximized and, thus, the developed 3-ion ICRF schemes can be applied to optimize the D-T fusion rate and achieve high neutron rate at moderate input heating power at JET (in a similar way as in $\mathrm{D}-{ }^{3} \mathrm{He}$ plasmas, see Fig. 12). Although the developed technique maximizes beam-target neutron rate in mixed plasmas and thus is not ITER-relevant, it facilitates the design of a dedicated scenario at JET, aiming to demonstrate alpha particle heating in the future D-T campaign.

We finally note that the 3-ion ICRF schemes are also relevant for ITER during its non-active operations [43, 44]. Reaching H-mode and the development of ELM control techniques are one of the main priorities for ITER during Pre-Fusion Power Operation phases [45]. Recent encouraging experimental results at JET-ILW demonstrated a significant reduction in the $\mathrm{H}$-mode power threshold when a small amount of ${ }^{4} \mathrm{He}$ ions, $n\left({ }^{4} \mathrm{He}\right) / n_{\mathrm{e}} \approx 10 \%$, was added to hydrogen plasmas [46]. This finding motivated the ITER team to consider the use of $\mathrm{H}-{ }^{4} \mathrm{He}$ plasmas to widen the $\mathrm{H}$-mode operational space in predominantly hydrogen plasmas [43]. Equally important, this mix allows also the application of the 3-ion ICRF scheme with the off-axis heating of ${ }^{3} \mathrm{He}$ minority ions, $n\left({ }^{3} \mathrm{He}\right) / n_{\mathrm{e}}<1 \%$, as proposed in [44], capable of additionally delivering up to $20 \mathrm{MW}$ of heating power in $\mathrm{H}+10 \%{ }^{4} \mathrm{He}$ plasmas in ITER. This scheme has been recently successfully demonstrated for the first time on the ASDEX Upgrade tokamak [47] and is also foreseen to be studied in future hydrogen campaign on JET. 


\section{Summary and conclusions}

Direct electron heating with ICRF mode conversion scenario is an attractive scheme in present-day tokamaks to modify the electron to ion temperature ratio without introducing significant populations of ICRF-accelerated fast ions [10]. However, application of this ICRF scenario in larger machines as ITER can be associated with difficulties for the excited fast waves to tunnel through the ion-ion hybrid evanescence layer, unwanted strong reflections of the incoming wave from the IIH cutoff and low single-pass wave damping. The novel 3-ion ICRF schemes [11-16] offer the possibility to achieve regimes with high single-pass wave damping and dominant ion absorption in such mixed or multi-ion plasmas instead of electron mode conversion heating. The key idea behind this technique relies on extending the plasma composition beyond two thermal ion species and adapt the plasma composition such that an additional ('third') ion component fulfills the resonant waveparticle interaction condition, Eq. (1), in the close vicinity of the ion-ion hybrid layer(s) in mixed plasmas. The developed heating scheme features strong localized ion cyclotron damping by the 'third' ion population, even at low concentrations.

Following the originally proposed realization of the 3-ion ICRF scheme in plasmas including three ion species with a different charge-to-mass ratio [11], an alternative realization of this novel heating scenario was proposed in the following paper [12]. This development has increased the flexibility for the application of the novel ICRF scheme and illustrated a novel way (so far, mostly unexploited) for using fast ions, such as NBI ions or fusion products (usually considered as a parasitic effect), to resonate at the IIH layer(s) and absorb ICRF power in mixed plasmas.

The detailed results of JET experiments (pulses \#91255 and \#91256) reported in this paper confirm good efficiency of heating mixed H-D plasmas with the 3-ion ICRF scheme, using fast NBI ions as a minority. Acceleration of D-NBI ions to energies of at least $1.3 \mathrm{MeV}$ with ICRF was independently confirmed by a set of fast-ion diagnostics, including NPA, neutron and gamma-ray measurements. Strong core localization of RF power deposition and generation of fast $\mathrm{D}$ ions with the 3-ion $\mathrm{D}-\left(\mathrm{D}_{\mathrm{NBI}}\right)-\mathrm{H}$ scheme was achieved by adapting the plasma composition to locate the IIH layer in the plasma core and adjusting NBI and ICRF settings to align the Doppler-shifted fundamental resonance of the D-NBI ions with the IIH layer. An independent verification for the core localization of the fast-ion population in the reported experiments was inferred from the analysis of MHD mode localization. The presence of the ICRF-generated supra-thermal D population resulted in a strong enhancement of the D-D neutron rate. In view of possible applications of the developed scheme for maximizing the beam-target neutron rate in D-T plasmas on JET, the possibility to moderate the fast-ion energies, e.g., by decreasing the ratio $P_{\mathrm{ICRF}} / P_{\mathrm{NBI}}$, was demonstrated.

In JET, the 3-ion ICRF schemes tuned for an efficient generation of fast ions in the plasma core mimic the heating conditions expected in D-T plasmas in ITER. ITER-relevant heating scenario with strong core electron heating from $\mathrm{MeV}$-range fast ions has been recently established in mixed $\mathrm{D}^{3}{ }^{3} \mathrm{He}$ plasmas on JET using the 3 -ion $\mathrm{D}-\left(\mathrm{D}_{\mathrm{NBI}}\right)-{ }^{3} \mathrm{He}$ scheme (see Fig. 12). These plasmas allow also to benchmark state-of-the-art gyrokinetic simulations to get a better understanding of the role of fast ions on microturbulence and thus help to clarify the possible impact of alpha particles in future ITER plasmas [42]. The detailed transport analysis of $\mathrm{D}-{ }^{3} \mathrm{He}$ plasmas with a large fraction of fast ions generated by the 3-ion ICRF scheme (\#94701) and the comparison with the NBI-only heating (\#94704) is ongoing and the results will be reported elsewhere. 
As discussed in Section 4 of the paper, the 3-ion schemes extend the use of ICRF in fusion research. Possible applications range from generating fast ions, including $\mathrm{D}-{ }^{3} \mathrm{He}$ fusion-born alphas, to bulk ion heating in $\mathrm{D}-\mathrm{T}=50 \%-50 \%$ plasmas. Recent theoretical and experimental developments of the 3-ion ICRF schemes on JET and AUG tokamaks have benefited strongly from the progress in validating ICRF codes for modeling these scenarios and benchmarking modeling results with fast-ion measurements [16, 34, 48]. While further more detailed analysis work remains to be done, e.g., better understanding the interaction of the fast ions with the plasma, MHD modes and turbulence, the results obtained recently confirm the high efficiency of the novel 3-ion ICRF schemes for plasma heating and increase our confidence in extrapolating the application of these schemes to ITER.

Acknowledgements. This work has been carried out within the framework of the EUROfusion Consortium and has received funding from the Euratom research and training programme 2014-2018 and 2019-2020 under grant agreement No 633053. The views and opinions expressed herein do not necessarily reflect those of the European Commission.

\section{References}

[1] ITER Physics Expert Group on Energetic Particles, Heating and Current Drive, Nucl. Fusion 39, 2495-2539 (1999)

[2] C. Gormezano et al., Nucl. Fusion 47, S285 (2007)

[3] J.R. Wilson and P.T. Bonoli, Phys. Plasmas 22, 021801 (2015)

[4] J. Ongena et al., Plasma Phys. Control. Fusion 59, 054002 (2017)

[5] M. Porkolab, AIP Conf. Proc. 314, 99-127 (1994)

[6] J. Adam, Plasma Phys. Control. Fusion 29, 443-472 (1987)

[7] J.-M. Noterdaeme et al., Fusion Sci. Tech. 53, 1103-1151 (2008)

[8] M.-L. Mayoral et al., Nucl. Fusion 46, S550-S563 (2006)

[9] D. Van Eester et al., Plasma Phys. Control. Fusion 51, 044007 (2009)

[10] M.J. Mantsinen et al., Nucl. Fusion 44, 33-46 (2004)

[11] Ye.O. Kazakov et al., Nucl. Fusion 55. 032001 (2015)

[12] J. Ongena et al., EPJ Web. Conf. 157, 02006 (2017)

[13] Ye.O. Kazakov et al., Nature Physics 13, 973-978 (2017)

[14] Ye.O. Kazakov et al., Recent advances in ICRF heating of mixture plasmas: survey of JET and AUG experiments and extrapolation to JET-DT and ITER Preprint: 2018 IAEA Fusion Energy Conf. (Gandhinagar, India, 22-27 October 2018), EX/8-1 (2018)

https://conferences.iaea.org/indico/event/151/papers/5659/files/4827-Kazakov-IAEA-FEC2018-paper-v5.pdf

[15] Ye.O. Kazakov et al., Phys. Plasmas 22, 082511 (2015)

[16] M.J. Mantsinen et al., Proc. 46th EPS Conf. on Plasma Physics, O5.102 (2019)

[17] E. Joffrin et al., Nucl. Fusion 59, 112021 (2019) 
[18] K. Ida, Nucl. Fusion 59, 117001 (2019)

[19] O. Asunta et al., Comp. Phys. Comm. 188, 33-46 (2015)

[20] M. Ono, Phys. Fluids B 5, 241-280

[21] V. Kiptily et al., Plasma Phys. Control. Fusion 48, R59-R82 (2006)

[22] L.-G. Eriksson and T. Hellsten, Nucl. Fusion 29, 875-883 (1989)

[23] M.-L. Mayoral et al., Nucl. Fusion 54, 033002 (2014)

[24] V.G. Kiptily et al., AIP Conf. Proc. 988, 283 (2008)

[25] D. Moseev et al., Rev. Mod. Plasma Phys. 2, 7 (2018)

[26] C. Hellesen et al., Nucl. Fusion 58, 056021 (2018)

[27] M. Schneider et al., Nucl. Fusion 56, 112022 (2016)

[28] J. Eriksson et al., Nucl. Fusion 55, 123026 (2015)

[29] J. Eriksson et al., Plasma Phys. Control. Fusion 61, 014027 (2019)

[30] A.S. Jacobsen et al., Nucl. Fusion 55, 053013 (2015)

[31] M. Salewski et al., Nucl. Fusion 57, 056001 (2017)

[32] A.S. Jacobsen et al., Rev. Sci. Instrum. 88, 073506 (2017)

[33] J. Varje et al., Journal of Instrumentation 12, C11025 (2017)

[34] K. Kirov et al., 23rd RF Topical Conference, invited talk (2019)

[35] M. Nocente et al., Plasma Phys. Control. Fusion 62, 014015 (2020)

[36] L. Giacomelli et al., Physics Procedia 62, 124-128 (2015)

[37] M. Brambilla et al., Plasma Phys. Control. Fusion 41, 1 (1999)

[38] R. Bilato et al., Nucl. Fusion 51, 103034 (2011)

[39] T. Craciunescu et al., Rev. Sci. Instrum. 87, 013502 (2016)

[40] S. Hacquin et al., Plasma Phys. Control. Fusion 49, 1371-1390 (2007)

[41] L. Meneses et al., Fusion Engineering and Design 86, 552-555 (2011)

[42] J. Garcia et al., Phys. Plasmas 25, 055902 (2018)

[43] ITER Research Plan, report no. ITR-18-003 (2018)

[44] M. Schneider et al., EPJ Web. Conf. 157, 03046 (2017)

[45] B. Bigot et al., Nucl. Fusion 59, 112001 (2019)

[46] J.C. Hillesheim et al., Proc. $44^{\text {th }}$ EPS Conf. on Plasma Physics, P5.162 (2017)

[47] H. Meyer et al., Nucl. Fusion 59, 112014 (2019)

[48] A. Kappatou et al., Proc. 45th EPS Conf. on Plasma Physics, O2.105 (2018) 